\title{
Absorption et assimilation du nitrate et recyclage de l'azote organique chez les plantes : intérêt pour le colza
}

\author{
Jean-François MOROT-GAUDRY \\ Mathilde ORSEL \\ Céline DIAZ \\ François DANIEL-VEDELE \\ Céline MASCLAUX-DAUBRESSE \\ Laboratoire de nutrition azotée des plantes, \\ INRA, Versailles \\ $<$ morot@versailles.inra.fr>
}

\section{Introduction}

Le colza est une espèce d'intérêt pour l'agriculture européenne (1/4 de la production mondiale en 2004). II est cultivé essentiellement pour la production d'huiles utilisées pour l'alimentation (huiles riches en acides gras insaturés), l'industrie (huiles enrichies en acide érucique) et la production de biocarburants (diesters). En Europe, les surfaces cultivées en colza sont voisines de 4,5 millions d'hectares. Avec le développement du diester, elles devraient approcher les 10 millions d'hectares dans les années 2010. Afin d'adapter la culture du colza aux objectifs de production à venir, prenant en compte les nouveaux débouchés et les impératifs de protection de l'environnement, il est nécessaire d'améliorer la productivité de cette plante, tout en réduisant les intrants.

Un des moyens est d'augmenter son efficacité à assimiler et gérer l'azote au cours de sa croissance et de son développement, en particulier en réduisant au printemps et à la récolte, les pertes d'azote par les feuilles non complètement vidées de cet élément et en améliorant les capacités de remobilisation de l'azote des parties végétatives vers les siliques. L'azote reste en effet l'élément essentiel de la productivité végétale et le coût énergétique de la production des engrais azotés est l'un des postes budgétaires les plus élevés à la charge des agriculteurs. Ainsi, pour rendre le coût des biocarburants acceptable, il faut réduire les coûts de production des produits issus des plantes qui les produisent, en abaissant en par-

\begin{abstract}
Brassica napus (winter oilseed rape) is an important agricultural crop cultivated for oil, which can be used as an edible product or for industrial application, bioester for example. Despite the very high capacity of oilseed rape to take up nitrate, many authors have reported a very low recovery of nitrogen in field-grown crops whatever the level of $\mathrm{N}$ fertilizer applied.

In this manuscript we describe the main biochemical and molecular mechanisms involved in nitrate uptake, reduction, assimilation and $N$ recycling during the reproductive period to gain sufficient knowledge to determine the relative importance of environmental and genetic factors determining $N$ management in plants. This understanding will provide the necessary background for improvement of oilseed rape varieties.
\end{abstract}

Key words: Brassica napus, bioester, nitrogen, nitrate

ticulier la quantité d'engrais azotés, poste fortement consommateur d'énergie et donc de pétrole. De plus, par une réduction de la consommation des engrais azotés, la pollution des nappes phréatiques et de l'atmosphère, les risques de verse et de maladie du feuillage seront réduits.

Pour mener à bien cette opération, il nous faut approfondir nos connaissances sur la physiologie de cette plante et développer des outils et des ressources génétiques à partir desquels il sera possible d'améliorer ses performances en particulier par une meilleure gestion de l'azote.

Voyons comment au cours de ces dix dernières années nos connaissances sur le métabolisme azoté des plantes ont évolué et comment le développement conjoint de la physiologie, de la génétique et de la génomique ont permis d'acquérir des connaissances plus pointues et plus globales sur le fonctionnement de la plante. Ces progrès ont été réalisés tout d'abord sur la plante modèle Arabidopsis thaliana mais devraient être transférés rapidement au colza, plante très proche génétiquement.

Cet article fait un point rapide sur les connaissances acquises récemment dans deux domaines du métabolisme azoté : l'absorption ou/et le transport du nitrate par les racines et la remobilisation de l'azote protéique issu de la dégradation de la machinerie photosynthétique foliaire au cours du vieillissement. D'immenses progrès ont été réalisés ces cinq dernières années dans la compréhension des mécanismes moléculaires (transporteurs protéiques de nitrate par exemple) et de leur régu- lation. II en est de même du développement des nouveaux outils de la génétique, cartographie, marqueurs génétiques et moléculaires, identification de QTL (Quantitative Trait Loci), recherche de gènes candidats. Enfin, le développement récent des techniques de génomique fonctionnelle apporte de nouveaux outils qui vont faire progresser nos connaissances tant dans le domaine de la physiologie que dans le domaine de la génétique.

\section{Schéma général de la voie d'assimilation du nitrate chez les plantes}

Après son entrée dans la racine grâce à des transporteurs membranaires, le nitrate peut être réduit et assimilé, stocké transitoirement dans la vacuole, ou exporté par les vaisseaux du xylème vers les feuilles pour y être réduit (figure 1). Il est observé que le nitrate est réduit essentiellement dans les racines chez les arbres et dans les feuilles chez les plantes herbacées. Dans tous les cas, le nitrate est réduit en nitrite par la nitrate réductase (NR) et le nitrite est réduit en ammonium par la nitrite réductase (NiR). Ces réactions de réduction nécessitent du pouvoir réducteur $(N A D(P) H)$ pour la NR et de ferrédoxine réduite pour la NiR. La NR est cytosolique, la NiR est plastidiale [1] (figure 2). L'ammonium est un composé toxique à faible concentration qui ne peut pas s'accumuler dans les cellules végétales. Son assimilation ou sa réassimilation sont donc des nécessités vitales pour les cellules. Deux enzymes, la gluta- 


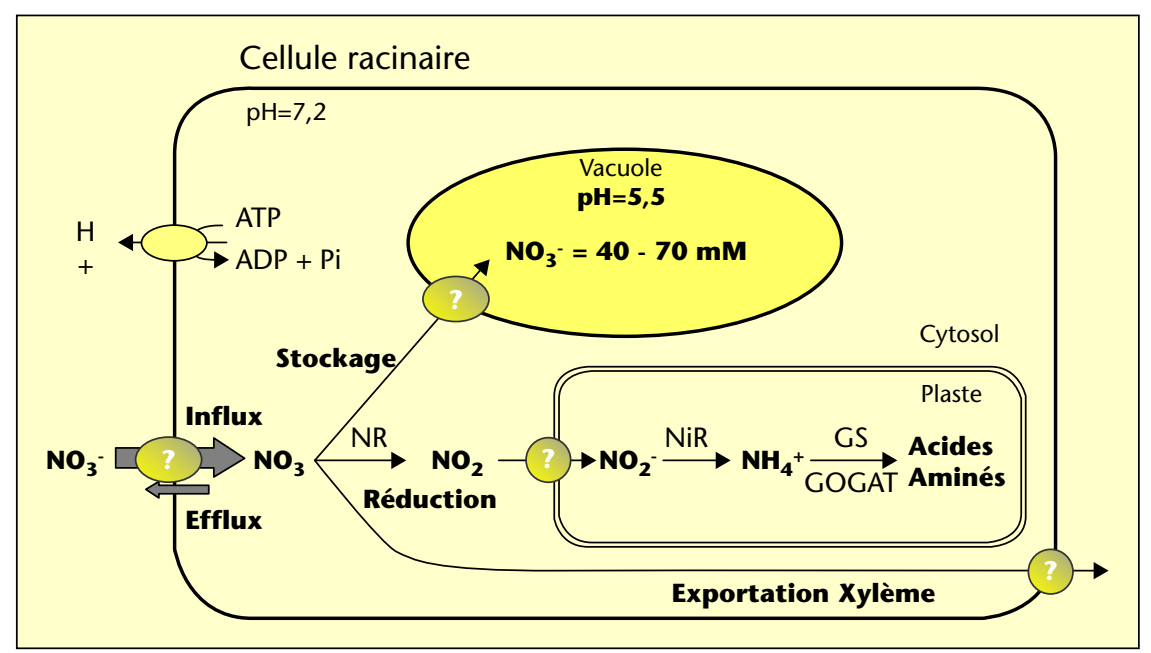

Figure 1. Schéma d'assimilation de l'azote chez les plantes : absorption racinaire du nitrate, stockage vacuolaire et réduction en nitrite et ammonium, synthèse des acides aminés et exportation; GOGAT, glutamate synthase; GS glutamine synthétase; NiR, nitrite réductase; NR, nitrate réductase. Sur la membrane plasmique qui entoure la cellule, sont localisés une pompe à protons $\left(\mathrm{H}^{+} /\right.$ATP ase) et un transporteur de nitrate (symport). (Thèse M Orsel, 2003).

mine synthétase (GS) et la glutamate synthase (GOGAT) catalysent le transfert de l'ammonium dans une molécule organique (figure 1). L'ion ammonium est incorporé au glutamate (Glu) par la glutamine synthétase (GS) pour former la glutamine (Gln). Le groupement amide de la glutamine est ensuite transféré à un acide cétonique, I' $\alpha$-cétoglutarate $(\alpha-K G)$ par la glutamate synthase (GOGAT). Deux molécules de glutamate sont ainsi formées, I'une est utilisée comme substrat de la glutamine synthétase (GS) pour la formation de glutamine, l'autre est source d'azote organique pour les réactions de transamination qui aboutissent à la synthèse de I'ensemble des acides aminés. La GS nécessite pour son fonctionnement de I'ATP, la GOGAT utilise le pouvoir réducteur de la ferrédoxine réduite [2].

Il existe deux isoenzymes de glutamine synthétase. La glutamine synthétase chloroplastique (GS2) est l'isoforme majoritaire des tissus photosynthétiques. Elle est associée à l'assimilation primaire du nitrate dans les feuilles et est impliquée dans l'assimilation de l'ammonium issu de la photorespiration. La glutamine synthétase cytoplasmique (GS1) est présente principalement dans les tissus non photosynthétiques. Elle joue entre autre un rôle important dans les

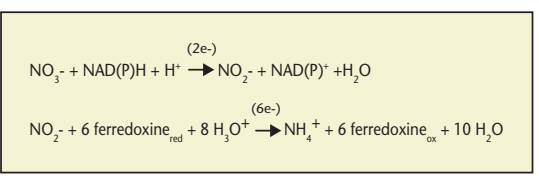

Figure 2. Activités nitrate et nitrite réductases. processus de remobilisation de l'azote pendant la sénescence, comme nous le verrons plus loin.

Enfin, une troisième enzyme est associée aussi au métabolisme de l'ammonium : la glutamate déshydrogénase (GDH). Il existe deux formes de $\mathrm{GDH}$, une forme majeure dépendante du $\mathrm{NADH}$ dans les mitochondries et une forme dépendante du NADPH dans les chloroplastes. L'enzyme est abondante dans les différents organes de la plante et son activité est généralement élevée. Cependant, son rôle physiologique est encore affaire de débat. En effet, son affinité très faible pour l'ammonium est incompatible avec un rôle majeur dans I'assimilation primaire de l'azote. II est supposé que la GDH jouerait plutôt un rôle prépondérant dans la désamination du glutamate et la formation $d^{\prime} \alpha$-cétoglutarate ; cette hypothèse est cohérente avec l'induction de l'activité observée pendant la germination et la sénescence, deux périodes pendant lesquelles le catabolisme des acides aminés est important [3].

Glutamine et glutamate sont donc les premiers acides aminés synthétisés après intégration de l'ammonium aux squelettes carbonés. Par transamination, l'azote est ensuite transféré à d'autres substrats carbonés (acides $\alpha$-cétoniques) pour former des nouveaux acides aminés (aspartate, alanine, glycine...) euxmêmes précurseurs de tous les autres acides aminés. Chez les végétaux, ces voies de synthèse, principalement chloroplastiques, sont complexes et très contrôlées [4].

\section{Absorption et transport du nitrate : aspect général}

Le nitrate et l'ammonium sont les principales sources d'azote pour la croissance des plantes dans la plupart des environnements naturels et agricoles. Le nitrate est considéré comme la source d'azote majoritaire, avec des concentrations 10 à 1000 fois plus élevées que celles de l'ammonium, notamment dans les sols bien aérés. Cependant, lorsque les deux éléments sont apportés aux mêmes concentrations, l'ammonium semble être la source d'azote préférentielle et son absorption est plus rapide que celle du nitrate.

Les plantes ont développé des mécanismes d'absorption complexes et fortement régulés pour assurer leur approvisionnement en azote dans les conditions de cultures les plus diverses. Nous décrirons essentiellement les mécanismes impliqués dans l'assimilation du nitrate.

\section{Absorption et transport du nitrate in planta}

L'azote étant absorbé au niveau racinaire sous forme de nitrate ou d'ammonium, les propriétés d'absorption et de transport et la capacité d'approvisionnement de la plante, sont directement reliées à la structure et à l'architecture du système racinaire (figure 3). Ainsi, la ramification importante de la racine principale par la formation de racines secondaires (ou latérales) augmente considérablement la surface d'échange entre le système racinaire et le sol. La différentiation de poils absorbants sur la couche épidermique (200 à $1000 / \mathrm{cm}^{2}$ ) est un facteur de ramification supplémentaire permettant l'optimisation du contact entre le sol et la racine.

Le transport des nutriments de l'eau du sol aux vaisseaux du xylème exige d'être à la fois sélectif et cumulatif. Les ions sont absorbés au niveau des cellules de l'épiderme racinaire ou rhizoderme, par un système de transport actif, c'est-à-dire qui se réalise dans le sens des potentiels électrochimiques croissants. Ils diffusent ensuite passivement, c'est-à-dire dans le sens des potentiels électrochimiques décroissants entre les différentes assises cellulaires du cortex racinaire, empruntant le réseau constitué essentiellement des parois cellulaires, c'est à dire la voie apoplastique. Cette diffusion est toutefois bloquée au niveau de l'endoderme où la paroi radiale des cellules est subérisée et donc rendue imperméable aux ions (bande de Caspary). Cette couche cellulaire qui isole le cortex du cylindre central (stèle) est un passage sélectif obligé qui nécessite le franchissement direct des membranes et milieux cellulaires (figure 3). Ce mode de diffusion à travers les différentes cellules est favorisé par la présence de plasmodesmes, ponts cytoplasmiques qui 


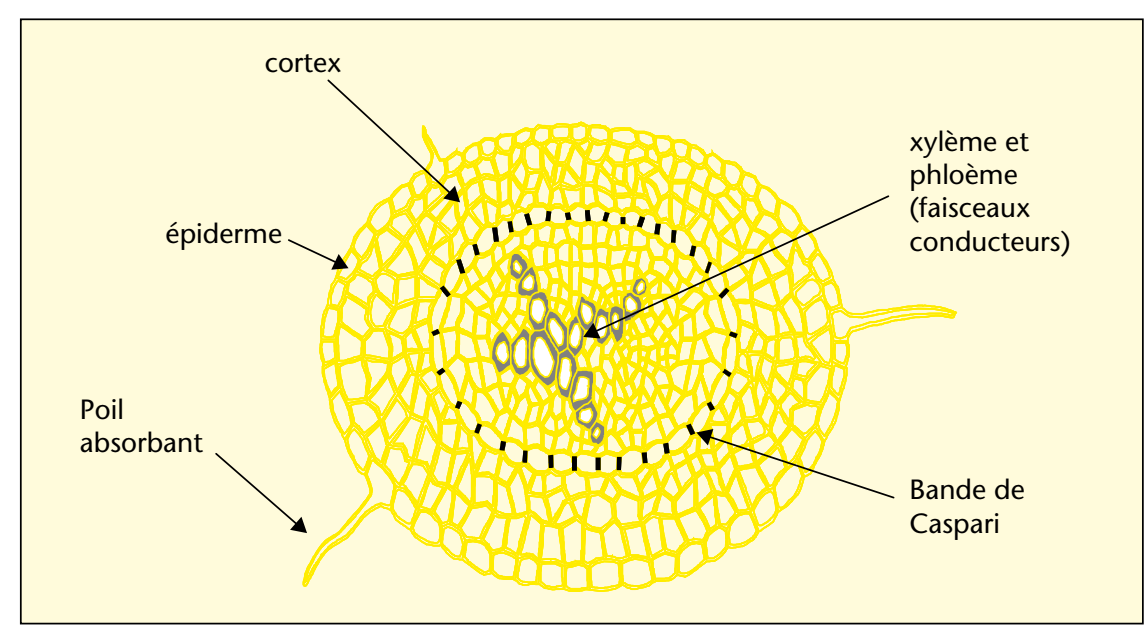

Figure 3. Coupe transversale de racine montrant les différents tissus : rhizoderme (ou épiderme racinaire) avec les poils absorbants, cortex, bande de Caspari et faisceaux conducteurs inclus dans la stèle ou cylindre central (Dessin Y. Roux).

forment ainsi un continuum cytoplasmique autorisant le passage des ions d'une couche cellulaire à l'autre par simple diffusion. Ce type de transport, voie symplastique, est plus rapide et plus efficace que la voie apoplastique (figure 3).

Après avoir été introduits dans le cylindre central, les ions nitrate sont exportés dans l'espace intercellulaire de la stèle jusqu'aux cellules parenchymateuses du xylème. Un système de transport actif permet à nouveau leur chargement souvent contre un gradient de concentration dans les vaisseaux du xylème où ils sont entraînés par le flux de sève dont le moteur est la transpiration. Chez les plantes herbacées, les ions nitrate sont généralement réduits dans les feuilles.

L'assimilation des ions nitrate (éléments polaires, hydrophiles) implique plusieurs franchissements de membranes biologiques: au niveau des poils absorbants, de la vacuole, des cellules de Caspary et du chargement dans le xylème. Le passage et l'échange de solutés, spécialement des ions, au travers de ces membranes lipidiques donc hydrophobes, sont extrêmement difficiles. Les membranes biologiques, en particulier le plasmalemme (membrane qui entoure la cellule) et le tonoplaste (membrane qui délimite la vacuole), constituent des barrières à la diffusion, empêchant le libre-échange des ions entre l'intérieur et l'extérieur de la cellule ou entre compartiments cellulaires. La présence de pompes et de transporteurs est donc nécessaire pour assurer le passage sélectif des ions au travers des membranes cellulaires et surtout pour vaincre les gradients de concentration en ions entre les différents compartiments cellulaires, ce qui nécessite une dépense d'énergie. (symport) et canaux ioniques (Dessin Y Roux).
Le transport d'un ion dans le sens croissant du gradient électrochimique nécessite une source d'énergie, le transport est dit actif (figure 4). Des systèmes spécifiques, des pompes à protons, fournissent cette énergie en hydrolysant des molécules très énergétiques, I'ATP. Chez les plantes, les $\mathrm{H}^{+}$/ATPases des membranes plasmiques et du tonoplaste sont les principaux moteurs du transport actif. Ces systèmes enzymatiques couplent I'hydrolyse de I'ATP à l'exportation des protons vers l'apoplasme (ensemble des parois) ou la vacuole et contribuent à la création et au maintien d'un gradient de protons et d'une différence de potentiel électrique de chaque côté de la membrane. Dans une cellule végétale type, le pH cytoplasmique est maintenu à environ 7,2 et celui de l'apoplasme à 5,5 ; la différence de potentiel électrique est de l'ordre de - $150 \mathrm{mV}$. Dans la vacuole, le pH est voisin de 5,5; la différence de potentiel électrique associée au tonoplaste est positive, de l'ordre de +20 à $+80 \mathrm{mV}$. De l'énergie est donc stockée sous forme de gradient électrochimique. Les ATPases pompes à $\mathrm{H}^{+}$créent donc à la fois une différence de potentiel électrique et une différence de $\mathrm{pH}$ de part et d'autre de la membrane, et donc une différence de potentiel électrochimique de $\mathrm{H}^{+}$. C'est cette différence, appelée gradient de potentiel électrochimique transmembranaire de $\mathrm{H}^{+}$, qui énergise la membrane et détermine le transport d'ions.

Des transporteurs (transporteurs protéiques d'anions ou de cations) utilisent cette énergie en couplant le retour spontané des protons au

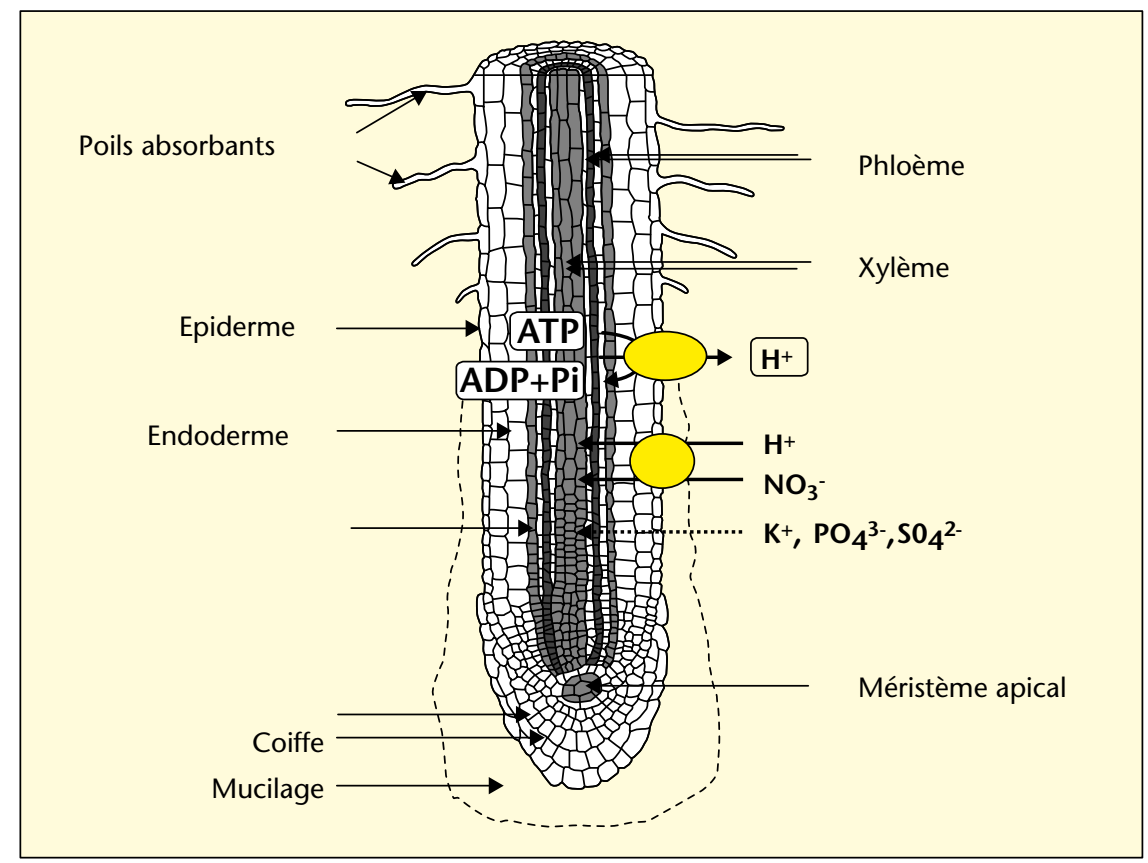

Figure 4. Coupe longitudinale de racine et mécanismes d'assimilation des ions, $\mathrm{H}^{+} /$ATP ase, transporteur de nitrate 
transport d'un autre soluté contre son gradient. Selon que les deux transports ont lieu dans le même sens ou en sens inverse, on distingue deux classes de transporteurs, respectivement les symports (même direction) et les antiports (directions opposées). II faut rappeler qu'un transporteur passe par un changement conformationnel à chaque fois qu'il transporte un substrat. La vitesse de transport est donc assez faible, de l'ordre de $10^{2}$ à $10^{3}$ éléments par seconde.

En résumé, on peut dire qu'un système de transport dit primaire, une $\mathrm{H}^{+}$/ATPase, couple I'hydrolyse de l'ATP à une excrétion de protons (création d'un gradient de $\mathrm{pH}$ ) et à une modification du potentiel électrique de part et d'autre de la membrane du compartiment cellulaire. La dissipation du gradient de protons entraîne le fonctionnement d'un système de transport dit secondaire, symport ou antiport. Enfin, si on considère les charges électriques, le transport peut être électroneutre (aucune charge nette n'est transportée) ou électrogène (une charge nette est transportée).

Quand le transport des ions se fait dans le sens des potentiels électrochimiques décroissants, c'est-à-dire qu'il s'effectue spontanément sans apport d'énergie, il peut être également assuré par des canaux (figure 4). Les canaux sont des systèmes protéiques, spécifiques du transport $d^{\prime}$ ions (canaux chlore, potassiques, etc.) et souvent unidirectionnels. Ils passent d'un état inactif (fermé) à un état actif (ouvert). La vitesse de transfert $d^{\prime}$ un canal est très élevée $\left(10^{7}\right.$ ions transportés par seconde), 1000 fois plus élevée que celle d'un transporteur protéique. II faut rappeler toutefois que la notion de canal et de transporteur n'est pas si tranchée qu'il ne parait. Des données récentes acquises sur le canal à chlore CCa montrent qu'un canal peut fonctionner également comme un transporteur [5].

In planta, le potentiel négatif de la membrane plasmique de la racine favorise l'entrée passive d'ammonium dans la cellule. En revanche dans le cas du nitrate, le potentiel négatif de la membrane et le gradient croissant de concentration ne permettent pas l'entrée passive du nitrate dans le cytoplasme et un système de transport actif est nécessaire. La caractérisation électrophysiologique des systèmes d'absorption du nitrate et de l'ammonium a permis de mettre en évidence différents systèmes de transport.

\section{Les systèmes d'absorption ou de transport du nitrate}

La concentration en nitrate des sols peut varier de plusieurs ordres de grandeur, de quelques micromolaires $(\mu \mathrm{M})$ à plusieurs millimolaires $(\mathrm{mM})$. Les plantes ont ainsi développé des sys-

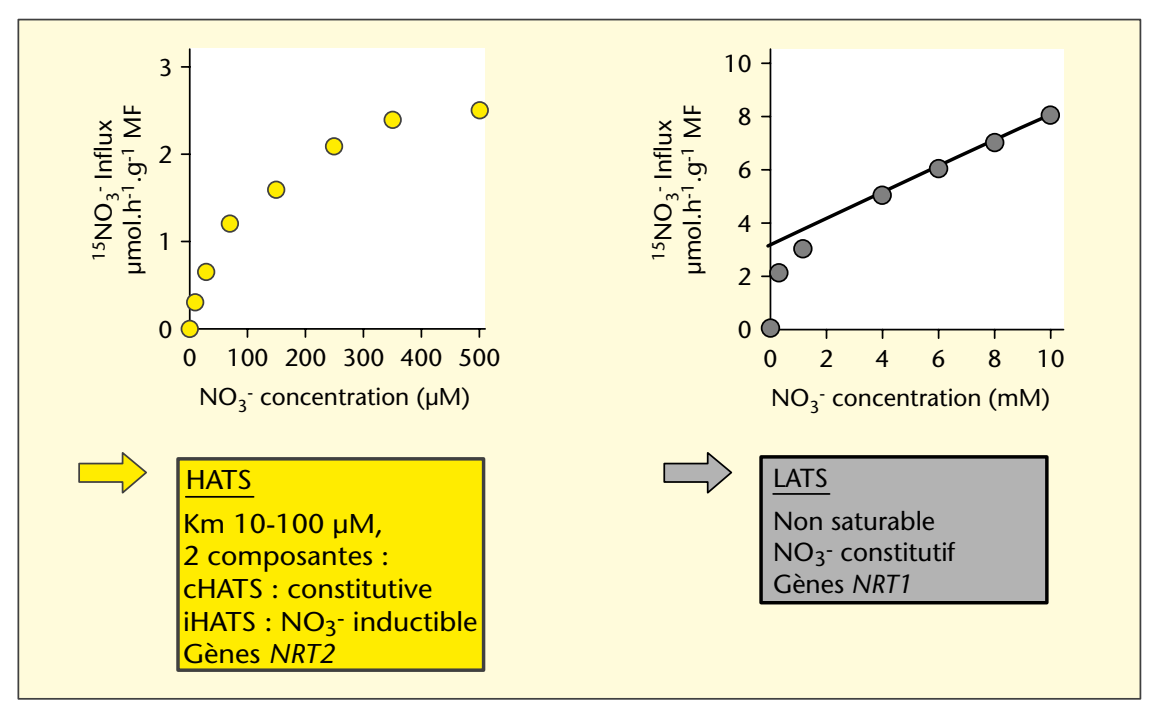

Figure 5. Cinétique d'assimilation du nitrate : système à haute affinité (HATS), constitutif et inductible, système à basse affinité (LATS) constitutif (Thèse M. Orsel, 2003).

tèmes d'absorption adaptés aux différentes concentrations rencontrées. Les études des cinétiques d'absorption du nitrate marqué $\left({ }^{15} \mathrm{NO}_{3}{ }^{-}\right)$en fonction de la concentration en nitrate externe ont mis en évidence dans les racines deux systèmes de transport, I'un à haute et l'autre à basse affinité (figure 5).

\section{Un système de transport à haute affinité (HATS)}

Pour des concentrations inférieures à 0,2$500 \mu \mathrm{M}$ de nitrate extérieur, l'influx de nitrate est essentiellement assuré par un système de transport à haute affinité, saturable au-delà de $200 \mu \mathrm{M}$ (figure 5). La particularité de ce système est de présenter deux composantes: I'une constitutive (CHATS) et l'autre inductible (iHATS). En effet, lorsque les plantes n'ont jamais été cultivées en présence de nitrate, I'activité du HATS est caractérisée par une très forte affinité pour le nitrate ( $\mathrm{Km}$ de 6 à $20 \mu \mathrm{M})$, mais une faible capacité de transport (Vmax de 0,13 à $1,3 \mu \mathrm{mol} \mathrm{h}^{-1} \mathrm{~g}^{-1}$ de matière fraîche), il $s^{\prime}$ agit de la composante constitutive du HATS. Lorsque les plantes sont mises en présence de nitrate, une deuxième composante du transport est induite, la capacité de transport du HATS est alors multipliée par 50. La composante inductible du HATS est caractérisée par une affinité pour le nitrate plus faible (Km de 13 à $79 \mu \mathrm{M})$ mais une capacité de transport supérieure (Vmax à $9,41 \mu \mathrm{mol} \mathrm{h}^{-1} \mathrm{~g}^{-1}$ de matière fraîche). II a été proposé que le système constitutif participe au maintien de l'homéostasie cellulaire. II permettrait à la concentration de nitrate cytoplasmique, régulée par la concentration de nitrate vacuolaire (rôle tampon de la vacuole), d'atteindre un seuil nécessaire à I'induction du second système de transport, le système inductible (iHATS). Les plantes disposeraient alors, pour de faibles concentrations en nitrate extérieur, d'un système d'absorption avec une forte capacité, donc très efficace [6].

\section{Un système de transport à basse affinité (LATS)}

Au-delà de 200-500 $\mu \mathrm{M}$ de nitrate externe, un système de transport à faible affinité entre en jeu (figure 5). Ce système est non saturable jusqu'à $50 \mathrm{mM}$ de nitrate. II est quantitativement majoritaire pour l'absorption du nitrate à partir de $1 \mathrm{mM}$. II peut être considéré comme non inductible par le nitrate, mais est cependant sujet à certaines régulations [6].

\section{Énergétique du transport}

L'utilisation d'inhibiteurs métaboliques a montré que les systèmes de transport du nitrate à haute et basse affinité nécessitent de l'énergie. Le coût énergétique global de l'absorption du nitrate chez l'orge avoisine 1 à 3 moles d'ATP pour une mole de nitrate absorbé, soit $10 \%$ à $20 \%$ du coût énergétique total de l'assimilation du nitrate.

Le mécanisme envisagé est un système de transport actif secondaire, comme défini précédemment. L'absorption du nitrate entraîne en général une alcalinisation du milieu extérieur, ce qui suggère que des protons pénètrent dans la cellule en même temps que les ions $\mathrm{NO}_{3}{ }^{-}$, ou bien que ces derniers sont échangés contre des ions $\mathrm{OH}^{-}$, ce qui revient au même. Les études électrophysiologiques ont montré de plus que le transport de nitrate est associé à une dépolarisation membranaire, le système est donc électrogène. II est admis que l'absorption du nitrate est due à un 
cotransport $\mathrm{H}^{+} / \mathrm{NO}_{3}{ }^{-}$(symport) dont la stœchiométrie serait de $2\left(\mathrm{H}^{+}\right): 1 \mathrm{NO}_{3}{ }^{-}[6]$.

\section{Régulation des systèmes de transport}

L'absorption du nitrate par les plantes qui implique la présence des systèmes de transport notamment dans la membrane externe (plasmalemme) ou vacuolaire (tonoplaste) des cellules racinaires, est un processus fortement régulé par la disponibilité en nitrate et ammonium et par le statut nutritionnel de la plante.

\section{Régulation par la disponibilité} du substrat azoté

La régulation de l'absorption du nitrate est induite par l'ion nitrate lui-même. L'influx à haute affinité du nitrate est transitoirement augmenté lorsque les plantes sont mises en présence de nitrate ; la composante inductible du HATS est induite par la présence de nitrate externe. La cinétique de l'induction est variable selon les espèces; le maximum d'influx est obtenu après $4 \mathrm{~h}$ à $6 \mathrm{~h}$ pour le maïs, $24 \mathrm{~h}$ pour I'orge et $48 \mathrm{~h}$ pour $A$. thaliana. L'utilisation $d^{\prime}$ 'inhibiteurs de la synthèse des ARNm et des protéines a mis en évidence que l'induction du iHATS implique la synthèse de nouveaux transporteurs protéiques.

L'ammonium, deuxième source d'azote minéral, interagit avec les systèmes d'absorption du nitrate lorsqu'il est présent dans le milieu de culture. À faible concentration, I'ammonium a un effet inducteur rapide. La stimulation de I'absorption du nitrate observée dans ce cas est attribuée au fait que l'absorption de l'ammonium entraîne l'acidification du milieu de culture (modification des gradients de protons), ce qui favorise le transport du nitrate. En revanche, à concentration plus élevée, l'ammonium inhibe rapidement et réversiblement l'absorption du nitrate, suggérant un effet direct de l'ammonium sur la membrane plasmique, modifiant en conséquence l'influx et parfois peut-être l'efflux de nitrate. L'inhibition par l'ammonium concerne principalement la composante inductible du HATS [6].

\section{Régulation par le statut nutritionnel de la plante}

Plusieurs observations ont permis de mettre en évidence la régulation de l'absorption du nitrate par le statut nutritionnel de la plante. Ces processus régulateurs coordonnent l'absorption du nitrate au développement des plantes. En effet, la capacité d'absorption du nitrate augmente au cours du développement végétatif ; elle est beaucoup plus élevée chez des plantules de soja âgées de 3 semaines que chez des plantules de 5 jours.

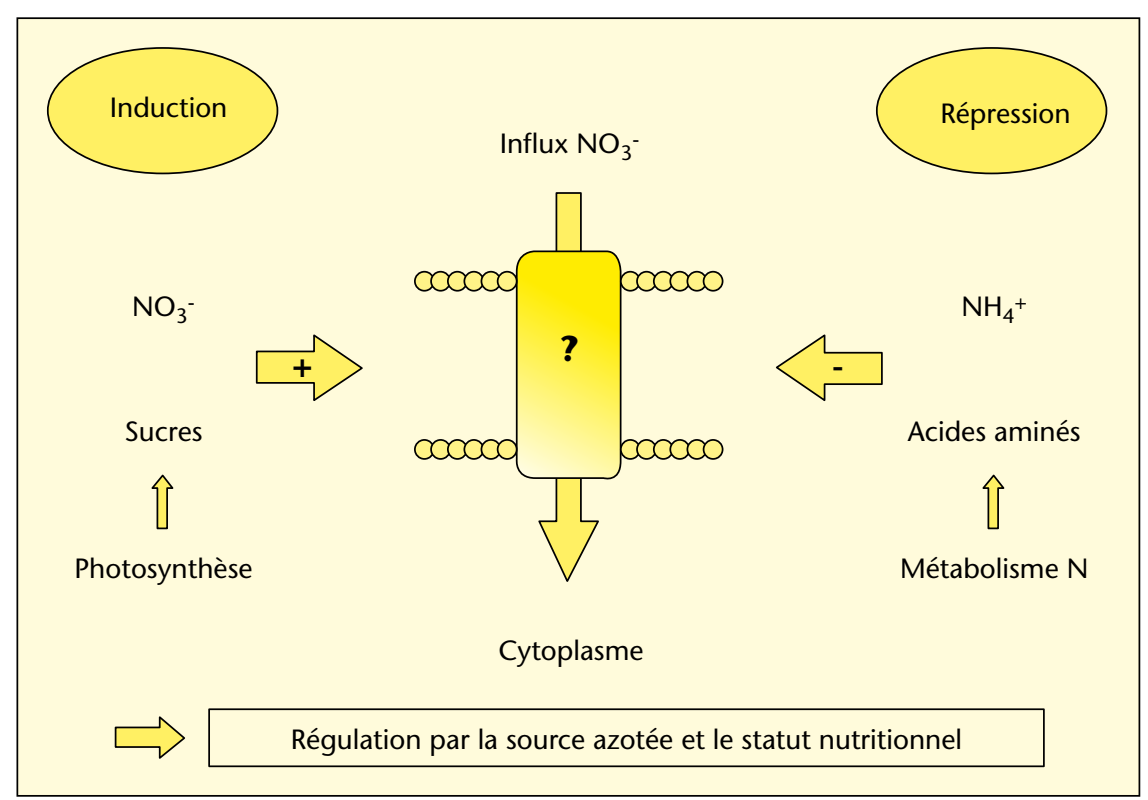

Figure 6. Mécanismes de régulation du transporteur de nitrate par les métabolites cellulaires (Dessin F. DanielVedele).

Peu d'éléments sont connus sur les signaux endogènes pouvant provoquer la réponse du système de transport au statut azoté de la plante. Une hypothèse communément admise est que le système d'absorption racinaire est sous le rétrocontrôle négatif de l'ion qu'il transporte (figure 6). L'effet inhibiteur du pool de nitrate interne sur sa propre absorption a pu être mis en évidence chez l'orge. En effet, lorsque des inhibiteurs de la voie d'assimilation du nitrate sont appliqués dans le milieu de culture, le nitrate s'accumule dans les plantes et sa vitesse d'absorption diminue. II en est de même chez des mutants qui accumulent le nitrate parce qu'ils sont déficients pour l'activité nitrate réductase [6].

Les acides aminés, produits lors de l'assimilation de l'azote, sont aussi de bons candidats pour jouer le rôle d'un signal « intégrateur » de la demande générale en azote de la plante. II a en effet été montré que la circulation des acides aminés est intense et rapide chez les plantes. D'autre part, après quelques heures d'incubation, I'addition d'acides aminés exogènes inhibe I'absorption du nitrate chez A. thaliana, le soja et le blé (figure 6) [6].

Les glucides peuvent également coordonner I'absorption du nitrate avec la photosynthèse et le métabolisme carboné. En effet, l'absorption nette du nitrate est soumise à des variations journalières; elle augmente continuellement au cours de la période lumineuse et décroît à l'obscurité. Des expériences réalisées sous des teneurs en $\mathrm{CO}_{2}$ faibles, réduisant ainsi I'activité photosynthétique, ont montré que l'absorption du nitrate est dépendante de la photosynthèse. Les deux processus étant phy- siquement séparés, cette réponse nécessite un signal coordinateur circulant entre la partie aérienne et le système racinaire. Les glucides, produits photosynthétiques, pourraient remplir ce rôle coordinateur (figure 6) [6].

\section{Bases moléculaires du transport du nitrate}

L'étude des bases moléculaires du transport du nitrate chez les plantes supérieures fait l'objet de nombreuses recherches depuis quelques années. Sur la base d'homologies de séquences, deux familles de gènes codant des transporteurs de nitrate ont ainsi été identifiées : les gènes NRT1 et NRT2. Ils codent des protéines appartenant respectivement aux familles PTR (peptide transporter) et NNP (nitrate-nitrite porter). Les deux familles de gènes se rencontrent à la fois chez les eucaryotes et les procaryotes. Ils appartiennent à la Major Facilitating Superfamily $[7,8]$ et codent des protéines qui partagent la même structure, avec douze domaines transmembranaires partagés en deux groupes de six hélices reliés par un domaine cytosolique.

\section{La famille NRT1}

Le premier gène de la famille, AtNRT1.1, a été isolé grâce à un mutant $\mathrm{d}^{\prime}$ insertion $\mathrm{d}^{\prime} A$. thaliana résistant au chlorate, analogue toxique du nitrate. Le mutant (chl1) présente une absorption réduite du nitrate à des concentrations supérieures à $1 \mathrm{mM}$ dans le milieu extérieur. Les études menées sur ce mutant suggèrent que AtNRT1.1 code un transporteur de nitrate à 
basse affinité à l'origine de l'absorption racinaire du nitrate.

Plusieurs autres membres de la famille NRT1 ont ensuite été identifiés par homologie chez A. thaliana, la tomate, le colza, Nicotiana plumbaginifolia et le riz. Jusqu'à présent, dans chacune des espèces étudiées, les gènes NRT1 forment une famille multigénique. L'analyse du séquençage complet $d^{\prime} A$. thaliana a révélé la présence de plus de 50 gènes NRT1. Le pourcentage d'identité entre les différentes protéines NRT1 est très variable. II est démontré également que les protéines NRT1 n'ont pas les mêmes propriétés de transport.

Les gènes NRT1 sont exprimés dans la partie racinaire, ce qui est compatible avec leur rôle supposé dans l'assimilation du nitrate. Chez $A$. thaliana (At), les deux gènes AtNRT1 sont exprimés principalement dans les racines, les ARNm étant localisés dans le rhizoderme et à la pointe racinaire. Dans les parties plus âgées de la racine, AtNR1.1 est exprimé dans le cortex et I'endoderme. L'expression de AtNRT1.2 a également été observée dans les poils absorbants. Le transcrit du gène AtNRT1.1 a été détecté à la pointe de la racine principale et des racines latérales, dans les jeunes feuilles, les boutons floraux en développement et les cellules de garde des stomates. L'analyse du mutant chl1 a permis de montrer que le transporteur de nitrate AtNRT1.1 joue un rôle déterminant dans la croissance des organes en développement et l'ouverture stomatique [6].

\section{La famille NRT2}

Les premiers membres de la famille NRT2 ont été identifiés chez les eucaryotes inférieurs. Le gène CRNA a été identifié chez le champignon Aspergillus nidulans à partir d'un mutant isolé sur la base de sa résistance au chlorate. L'expression de la protéine dans les ovocytes de xénope a permis de montrer que CRNA code un transporteur de nitrate/nitrite. Deux autres gènes, CrNRT2.1 et CrNRT2.2, ont ensuite été identifiés chez des mutants de délétion de I'algue verte Chlamydomonas reinhardtii, déficients dans le transport du nitrate et/ou du nitrite. Un transporteur à haute affinité du nitrate, YNT1, a également été cloné chez la levure Hansenula polymorpha à partir d'un mutant déficient pour l'absorption du nitrate à faible concentration.

La comparaison des séquences d'acides aminés des transporteurs de nitrate/nitrite des algues et des champignons a révélé des régions très fortement conservées qui ont permis d'identifier $d^{\prime}$ autres membres de la famille de transporteurs sur la base de leur homologie de séquence. La plupart des membres de la famille NRT2 des plantes supérieures ont ainsi été identifiés chez l'orge, N. plumbaginifolia, $A$. thaliana, le soja, et la tomate. Pour chacune des espèces étudiées, les gènes NRT2 forment une famille multigénique : au moins deux gènes chez N. plumbaginifolia et jusqu'à sept ou dix gènes chez l'orge. L'analyse du génome complet $d^{\prime} A$. thaliana a révélé une famille multigénique de sept membres de gènes NRT2.

Les gènes de la famille NRT2 codent des protéines à douze segments transmembranaires de $60 \mathrm{kDa}$ environ. Un motif fortement conservé (A-G-W/L-G-N-M-G) dans le cinquième domaine transmembranaire serait la signature de la famille. Les membres de la famille peuvent être séparés en trois groupes sur la base de leur séquence en acides aminés: les procaryotes, les champignons, les algues et les plantes.

Les caractéristiques d'expression des gènes NRT2 permettent de penser qu'ils sont bien impliqués dans le système de transport iHATS. En effet, ils sont tous exprimés préférentiellement dans les racines; des expériences $d^{\prime}$ hybridation in situ ont mis en évidence I'expression préférentielle du gène NpNRT2.1 dans les primordia des racines latérales et le rhizoderme des racines matures. Les premiers gènes NRT2 isolés ont une expression induite par le nitrate et réprimée par des sources d'azote réduit comme l'ammonium ou la glutamine. Le gène AtNRT2.1 a d'ailleurs été identifié pour la première fois sur la base d'une forte induction par le nitrate par rapport à la glutamine. Enfin, l'influx du nitrate est fortement corrélé avec l'expression des gènes NRT2. La cinétique d'induction par le nitrate de l'expression du gène AtNRT2.1 est parallèle à l'induction transitoire de l'activité du système de transport iHATS; cette corrélation a depuis également été observée chez l'orge. De même, lors d'une carence en azote, l'induction transitoire de l'expression de AtNRT2.1 correspond à I'induction transitoire de l'influx à haute affinité du nitrate. Le niveau d'expression d'AtNRT2.1 est également corrélé aux variations journalières de l'absorption nette du nitrate : I'addition de saccharose dans le milieu de culture maintient l'expression de AtNRT2.1 et empêche la chute de l'activité du HATS à l'obscurité [9]. M. Orsel [9] a réalisé une analyse phylogénétique de la famille des transporteurs NRT2 et a étudié les caractéristiques d'expression de chacun des gènes en fonction des organes et $d u$ statut azoté des plantes. Elle a observé des caractéristiques différentes entre chacun de ces transporteurs, suggérant un rôle spécifique pour chacun des transporteurs de la même famille. Afin d'obtenir une preuve fonctionnelle du rôle des gènes AtNRT2 dans le système de transport HATS, un mutant atnrt2.1a, spécifiquement affecté pour l'un de ces gènes, a été identifié par une stratégie de génétique inverse. II s'agit en fait d'un double mutant pour les gènes AtNRT2.1 et AtNRT2.2, spécifiquement modifié dans la composante inductible du système HATS. De plus, une analyse physiologique du mutant a mis en évidence le rôle important des gènes AtNRT2.1 et/ou AtNRT2.2 dans la croissance des plantes, cultivées sur une source de nitrate limitant $(0,2$ $\mathrm{mM}$ ) (figure 7). Enfin, les propriétés de transport du nitrate par les protéines AtNRT2 sont étudiées dans le système d'expression hétérologue (ovocyte de xénopes).

Un faisceau d'indices concordant autorise à penser que les gènes NRT2 des plantes supérieures codent des transporteurs à haute affinité du nitrate. Cependant, aucune preuve fonctionnelle $n^{\prime}$ est encore disponible $[10,11]$. La présence d'une famille multigénique dans chaque organisme étudié pose le problème de la redondance ou de la spécificité de la fonction de chacune des protéines de la famille NRT2 dans la nutrition azotée des plantes [9].

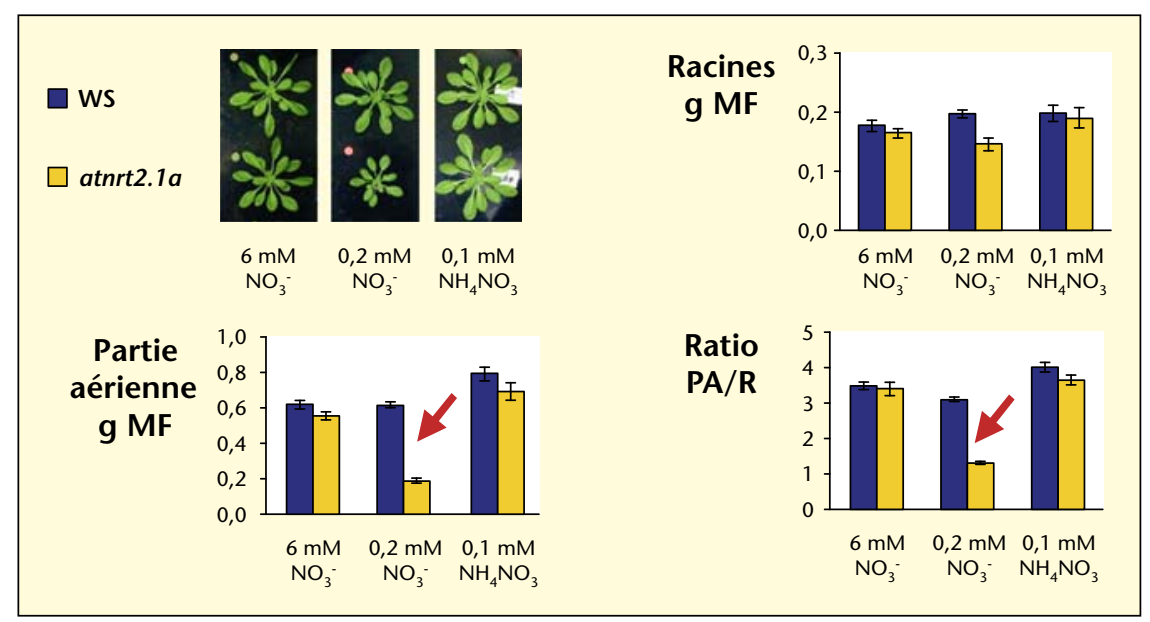

Figure 7. Effets de la nutrition azotée sur la croissance (MFparties aériennes, racines et rapport partie aérienne/racines) d'Arabidopsis thaliana sauvages (ws) et transformés (atnrt2.1a) cultivées sur nitrate 0,2, $6 \mathrm{mM}$ et sur nitrate d'ammonium 0,1 mM (Thèse M Orsel, 2003 ; Orsel et al., Planta 2004 ; 219 : 714-721). 
Cependant, une approche de génétique inverse a montré qu'un double mutant, affecté dans l'expression des gènes AtNRT2.1 et AtNRT2.2, présente un défaut dans le iHATS mais pas dans le LATS $[10,11]$.

Les études sur les transporteurs de nitrate se poursuivent. II a été démontré chez Chlamydomonas, algue unicellulaire, que pour obtenir un transport efficace du nitrate, il était nécessaire que deux gènes soient impliqués, le gène NRT2 qui code le transporteur transmembranaire à 12 éléments, et un second gène, le gène CrNar2 qui code une petite protéine de 200 résidus d'acides aminés. Ces deux gènes existent chez les plantes. La présence de ces deux gènes pour un fonctionnement efficace $d u$ transport haute affinité du nitrate a été confirmée par Tong et al. [12] et Okamoto et al. [13-15]. II a été observé également que les transporteurs de nitrate étaient impliqués non seulement dans la régulation des flux de nitrate mais aussi dans la régulation de la croissance cellulaire, en particulier dans le développement des racines latérales $[8,16]$.

\section{Transport à longue distance des acides aminés}

Le transport à longue distance des acides aminés est réalisé soit par la sève du xylème quand ils sont synthétisés dans les racines, soit par la sève du phloème quand ils sont synthétisés dans les feuilles.

Les tissus du xylème sont constitués de longs tubes vides dont la paroi présente des épaississements lignifiés. Ces éléments morts conduisent la sève brute ou xylémienne des racines vers les feuilles. Les ions sont entraînés par le flux de sève dont le moteur est la transpiration. Les tissus du phloème sont constitués d'éléments conducteurs, les tubes criblés, associés à des cellules parenchymateuses, les cellules compagnes. Les tubes criblés sont des cellules vivantes allongées mais dépourvues de noyau, connectées par des cribles. Les cellules compagnes jouent un rôle important dans le chargement du phloème en saccharose et acides aminés. Le complexe phloémien constitué des tubes criblés et des cellules compagnes transporte les acides aminés des feuilles aux autres organes de la plante.

Le chargement des acides aminés dans le système phloémien correspond à un transport actif secondaire, comparable à celui observé dans le transport du nitrate. C'est un transport de composés chargés contre un gradient de concentration, ce qui entraîne une dépense d'énergie. Un système de transport primaire, une ATPase à protons couple l'hydrolyse d'ATP à une excrétion de protons. Son fonctionnement crée un gradient de $\mathrm{pH}$ et une différence de potentiel électrique de chaque côté de la membrane. Ces déséquilibres entraînent le fonctionnement de systèmes de transports secondaires, des symports et des antiports, les transporteurs d'acides aminés. Ces dernières années, des dizaines de transporteurs d'acides aminés ont été identifiées. Ils sont spécifiques de tissus et d'organes mais pas toujours spécifiques d'un acide aminé ou d'un groupe d'acides aminés donnés [17].Une fois chargés dans les tubes criblés du phloème, les composés sont entraînés par le flux de masse et distribués à tous les organes de la plante. Le flux de masse résulte de la différence de concentration en composés entre le lieu de chargement et de déchargement et des mouvements d'eau qui l'accompagnent [18].

\section{Mobilisation de l'azote organique au cours du développement des plantes}

Nous terminerons notre article par une étude rapide des mécanismes mis en jeu dans la remobilisation de l'azote organique lors du vieillissement des feuilles.

\section{Sénescence et remobilisation} de l'azote, dans les feuilles

Pendant la phase de croissance végétative, les feuilles et racines en développement absorbent et assimilent le nitrate et l'ammonium et incorporent l'azote aux molécules carbonées pour former les acides aminés, précurseurs des protéines, et acides nucléiques, supports de l'information génétique. Dans les feuilles photosynthétiques l'azote est généralement retrouvé dans la machinerie photosynthétique sous la forme de photosystèmes et d'enzymes comme la Rubisco (enzyme de carboxylation et d'oxygénation qui représente à peu près $50 \%$ des protéines foliaires solubles). Certaines plantes peuvent stocker également l'azote sous forme de protéines plus spécifiques, appelées VSP (Vegetative Storage Protein) utilisées comme réserve temporaire et transitoire d'azote.

À partir d'un stade de développement appelé maturité, les organes végétaux, les feuilles en particulier, entrent dans une phase de sénescence. La sénescence débute aux premiers signes de démantèlement des structures photosynthétiques et s'achève par la mort cellulaire. II est admis que la sénescence foliaire est un phénomène graduel qui ordonne dans le temps une succession d'évènements moléculaires, biochimiques et métaboliques. Cette succession d'événements est génétiquement codée. L'apparition de la sénescence peut être néanmoins précipitée ou ralentie en fonction des conditions environnementales et nutritives, mais elle reste inéluctable et conduit inexorablement à la mort de l'organe [18].
Au cours de la sénescence foliaire, le recyclage de l'azote s'intensifie. II constitue une étape essentielle dans la gestion de cet élément en permettant une réutilisation de l'azote dans les organes en croissance, jeunes feuilles ou fruit et grains. La remobilisation de l'azote constitue une économie considérable pour la plante et contribue largement à la constitution des organes récoltables puisque l'on estime que plus de la moitié de l'azote des grains de blé ou de maïs provient de la remobilisation foliaire. En contrepartie, la restitution au sol de l'azote organique non remobilisé, forme une source d'azote peu utilisable sans minéralisation préalable. Elle peut être à l'origine d'une pollution qui peut être préoccupante au niveau écologique ; c'est le cas du colza qui perd au cours de son développement des feuilles encore riches en azote. L'impact de la sénescence du point de vue agronomique concilie deux problèmes apparemment antinomique : poursuite du fonctionnement de la photosynthèse pour assimiler le maximum de carbone et nécessité de remobiliser l'azote réduit des feuilles pour la constitution des protéines des organes récoltables. Il est observé que l'augmentation des rendements est largement tributaire de la longévité de I'appareil photosynthétique et que la remobilisation de l'azote des feuilles sénescentes (démantèlement de l'appareil photosynthétique) est déterminante pour les teneurs en azote des grains. En sélectionnant de hauts rendements, ont donc été retenues des plantes dont les feuilles sont en nombre défini mais de longévité supérieure (le maïs), ou bien des écotypes qui renouvellent leurs feuilles dès que les premiers signes de sénescence apparaissent, ce qui assure une conservation de la capacité photosynthétique globale. Il est probable que cette sélection ait été faite au détriment de l'efficacité de la remobilisation de l'azote. La connaissance des mécanismes de réutilisation de l'azote foliaire et de leur régulation est essentielle à I'amélioration des produits de l'agriculture (contenu protéique des organes récoltables) et à la préservation des sols.

\section{Mobilisation de I'azote foliaire chez le tabac}

Des travaux [19] ont permis de montrer que les feuilles de la tige de tabac peuvent être classées en deux groupes. Les feuilles proches de l'apex sont appelées feuilles "puits» (jeunes ou matures), essentiellement dédiées aux fonctions d'assimilation primaire de l'azote et du carbone (figure 8). Dans ces feuilles, les gènes codant les enzymes impliquées dans les fonctions d'assimilation primaire de l'azote, nitrate et nitrite réductases (NR et NiR), glutamine synthétase et glutamate synthase chloroplastiques (GS2 et GOGAT) sont bien exprimés. Les feuilles proches du collet représentent en 


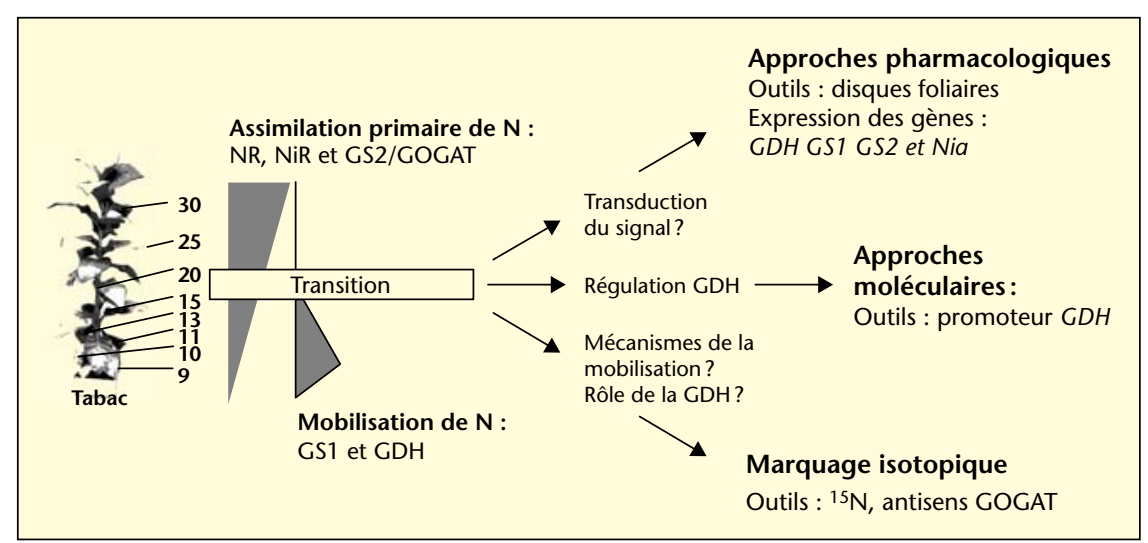

Figure 8. Mobilisation de l'azote dans les feuilles sénescentes de tabac (Nicotiana tabacum): GDH, glutamate déshydrogénase ; GOGAT, glutamate synthase ; GS1 et GS2 glutamine synthétases cytosolique et chloroplastique ; $N i R$, nitrite réductase; NR, nitrate réductase (gène Nia) (Dessin C Masclaux-Daubresse).

revanche les feuilles "sources» (matures et sénescentes), dans lesquelles s'expriment les gènes codant les enzymes glutamine synthétase cytosolique (GS1) et glutamate déshydrogénase (GDH). Ces deux enzymes sont généralement présentes dans les racines. GS1 et GDH seraient impliquées dans la gestion des pools de glutamate et glutamine des feuilles sénescentes. Ces enzymes, cytosoliques pour la première et mitochondriales pour la seconde, pourraient compenser, au cours du vieillissement, la perte des fonctions chloroplastiques GS2 et GOGAT (figure 8).

La transition d'un statut d'organe puits (section apicale) vers un statut d'organe source (section basale) s'accompagne donc de bouleversements métaboliques importants comme l'accumulation des sucres et l'épuisement des formes libres aminées et inorganiques de l'azote [19].

Afin de préciser les relations sources /puits au cours du vieillissement foliaire, des expériences utilisant des composés marqués par l'isotope lourd ${ }^{15} \mathrm{~N}$ (ammonium et glutamate) ont été réalisées sur des disques de feuilles sources et de feuilles puits. Les résultats obtenus ont montré que, quel que soit l'âge des feuilles, l'ammonium est assimilé par la voie GS/GOGAT. La $G D H$ est essentiellement impliquée dans les mécanismes de déamination, fournissant des squelettes carbonés au métabolisme.

Une étude comparative de l'expression différentielle des gènes $G D H, G S$ et $N R$ a également été réalisée chez le tabac. Un système d'induction in vitro sur disques foliaires a permis d'identifier des signaux (métaboliques, hormonaux ou de stress biotiques et abiotiques) qui contrôlent l'expression de ces gènes. Par une approche pharmacologique, les voies de transduction allant de la perception de ces signaux jusqu'à l'expression des gènes, ont été caractérisées $[20,21]$.

\section{Approche génétique de la mobilisation de l'azote}

Afin de mieux connaître les bases génétiques qui contrôlent les mécanismes de mobilisation de l'azote au cours de la sénescence foliaire, une approche génétique a été entreprise. La variabilité de marqueurs de sénescence et de mobilisation de l'azote a été réalisée sur une collection réduite de la population de 420 lignées recombinantes (Rils) issues du croisement entre les écotypes sauvages parentaux d'A.thaliana, Bay-0 et Shahdara [22].

Cette approche a permis de sélectionner les caractères présentant une variabilité significative au sein de la sous-population ainsi qu'une héritabilité suffisante pour envisager une étude QTL. Cinq QTL de jaunissement (destruction Diaz, 2005 ; Diaz et al., Plant Cell Physiol 2006 ; 47 : 74-83). des chlorophylles) et six QTL liés au rougissement (accumulation d'anthocyanes) ont été identifiés (figure 9). Le degré de jaunissement des feuilles qui a été mesuré à l'aide d'un logiciel d'analyse d'image a été choisi comme critère de sénescence. L'analyse du degré de jaunissement des feuilles a été effectuée sur deux cultures indépendantes de 300 lignées recombinantes. Les résultats obtenus ont été comparés avec des résultats issus de notations visuelles [23]. La colocalisation des QTL de sénescence et de QTL d'efficacité d'utilisation de l'azote NUE (teneurs en azote total, concentrations en acide aminés) a permis de montrer que les lignées les plus sénescentes sont celles qui ont la plus petite biomasse (masse sèche) et les plus fortes concentrations en azote et en acide aminés. Ceci montre que la vitesse de développement foliaire joue sans doute un rôle majeur dans l'efficacité d'utilisation de l'azote.

Suite à l'estimation du taux de jaunissement des rosettes, cinq lignées recombinantes ont été choisies pour leurs différences de sénescence : trois lignées sénescentes, deux lignées non sénescentes. La comparaison de ces lignées au cours de la cinétique de croissance a révélé de nouveaux marqueurs métaboliques de la sénescence foliaire et montré que l'expression des marqueurs de mobilisation de I'azote (GS1, GDH, protéolyse) augmentait au cours du vieillissement quels que soient les génotypes considérés et était corrélée au niveau de sénescence foliaire atteint dans chaque génotype. L'existence d'un point de rupture dans le développement des lignées suggère l'existence d'un "senseur» capable d'orienter le comportement métabolique des

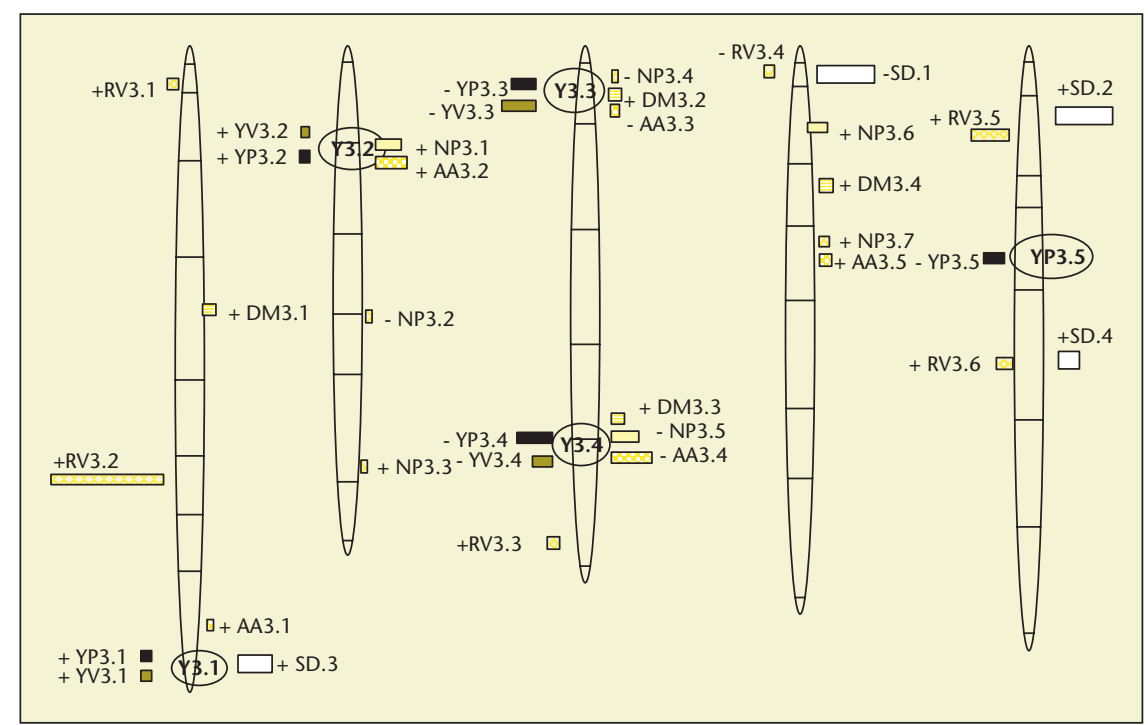

Figure 9. Identification de QTL associés au jaunissement et au rougissement de feuilles de lignées recombinants d'A. thaliana (Bay- $0 \times$ Shadara). Les QTL sont représentés par des barres; $A A$, teneur en acides aminés totaux; DM, matière sèche; $N P$, teneur en azote total ; $R$, rougissement ; $S D$, floraison en jours courts; $Y$, jaunissement. (Thèse $C$ 
tissus foliaires vers la remobilisation [24]. L'étude par génomique fonctionnelle des acteurs impliqués potentiellement dans la remobilisation de l'azote foliaire (notamment les GS1) se poursuit et devrait permettre de contrôler dans l'avenir le devenir de l'azote remobilisé pour une meilleure efficacité du remplissage du grain et également pour une meilleure économie de l'azote au niveau plante entière.

\section{Conclusion}

L'approche moléculaire de l'étude des transporteurs et des enzymes et des gènes correspondants permet d'approfondir nos connaissances des mécanismes intimes qui régissent le métabolisme azoté. Toutefois, ces approches très réductionnistes mais nécessaires doivent être complétées par des approches plus globales de l'ensemble des mécanismes, in planta, qui pourront fournir une vue plus intégrée de l'expression de l'ensemble des gènes concernés et des conséquences sur la régulation des voies d'assimilation et de gestion de l'azote chez le colza au cours de son développement [25-27].

L'existence de cartes génétiques de référence denses, construites à l'aide de marqueurs moléculaires (marqueurs microsatellites), rend désormais possible la localisation des zones chromosomiques impliquées dans la variation des caractères agronomiques (Quantitative Trait Loci ou QTL). En parallèle, I'établissement de cartes physiques basées sur l'ordonnancement à l'aide de diverses techniques de grands inserts d'ADN génomique permettra d'accélérer la localisation de gènes candidats pour le caractère en question.

Les approches génomiques, en apportant une vision plus intégrée sur le fonctionnement de la plante, fourniront de nouvelles données pertinentes en termes de fonction des gènes, de régulation en réseau des gènes et d'effets des différents allèles sur la variation d'un caractère. Elles permettront de mettre en place des stratégies de sélection assistée par marqueurs et de développer des modèles de prédiction du fonctionnement de la plante au champ pour une agriculture plus précise et plus durable. Enfin, le développement de la génomique comparative permettra d'accélérer l'acquisition de connaissances sur les caractères agronomiques importants en améliorant notamment le transfert de connaissances $\mathrm{d}^{\prime} A$ thaliana, la plante modèle, vers le colza tout en tenant compte des spécificités biologiques de cette plante oléagineuse très proche génétiquement. On peut espérer ainsi la mise sur le marché de variétés de colza qui géreront mieux l'azote et compatibles avec les contraintes industrielles, économiques et environnementales nouvelles.
Les apports de la génomique sont considérables. L'étude du transcriptome par les "puces» permet d'étudier simultanément l'expression de plusieurs milliers de gènes et $d$ 'identifier les gènes plus (ou moins) exprimés dans une condition que dans une autre (fort ou faible azote par exemple). L'étude du protéome et du métabolome donne des informations au niveau de la traduction en protéines, de leur fonctionnement conduisant à des compositions variables en métabolites. L'étude comparative à haut débit de mutants dans lesquels le gène n'est pas fonctionnel ou au contraire où il est exprimé constitutivement, fournit rapidement des informations sur sa fonction. Ces techniques commencent à être mises en œuvre avec succès chez bon nombre de plantes.

Remerciements. Les auteurs remercient Sylvain Chaillou et Michèle Cren pour la relecture de ce manuscrit.

\section{RÉFÉRENCES}

1. MEYER C, STITTM. Nitrate reduction and signalling. In : Lea P, Morot-Gaudry JF, eds. Plant. Nitrogen. INRA Springer Verlag, 2001 : 37-59.

2. HIREL BPJ, LEA PJ. Ammonia assimilation. In : Lea P, Morot-Gaudry JF, eds. Plant. Nitrogen. INRA Springer Verlag, 2001 : 79-99.

3. MASCLAUX C, QUILLERÉ I, GALLAIS A, HIREL B. The challenge of remobilisation in plant nitrogen economy. A survey of physio-agronomic and molecular approaches. An Appl Biol 2001 ; $138: 69-81$; (special issue).

4. MOROT-GAUDRY JF, D. JOB D, LEA PJ. Amino acid metabolism. In : Lea P, Morot-Gaudry JF, eds. Plant. Nitrogen. INRA Springer Verlag, $2001: 167-211$

5. DE ANGELI A, MONACHELLO D, EPHRITIKHINE G, et al. The nitrate/proton antiporter AtCLCa mediates nitrate accumulation in plant vacuoles. Nature 2006 ; 442 : 939-43.

6. TOURAINE B, DANIEL-VEDELE $F, \quad$ FORDE $B$. Nitrate uptake and its regulation. In : Lea $P$, Morot-Gaudry JF, eds. Plant. Nitrogen. INRA Springer Verlag, $2001: 1-36$.

7. ORSEL M, FILLEUR S, FRAISIER V, DANIELVEDELE F. Nitrate transport in plants: which gene and which control? J Exp Bot 2002; 53 : 825-33.

8. LITTLE D, RAO H, OLIVA S, DANIEL-VEDELE F, KRAPP A, MALAMY JE. The putative highaffinity nitrate transporter NRT2-I represses lateral root initiation in response to nutritional cues. Proc Natl Acad Sci USA 2005; 102 : 13693-8.

9. ORSEL M, EULENBURG $K$, KRAPP A, DANIELVEDELE F. Disruption of the nitrate transporter genes AtNRT2.1 andAtNRT2.2 restricts growth at low external nitrate concentration. Planta $2004 ; 219$ : 714-21.

10. CEREZO M, TILLARD P, FILLEUR S, MUNOS S, DANIEL-VEDELE F, GOJON A. Major alterations of the regulation of root $\mathrm{NO}_{3}{ }^{-}$uptake are associated with the mutation of NRT2.1 and NRT2.2 genes in Arabidopsis. Plant Physiol $2001 ; 127$ : 262-71.

11. FILLEUR S, DORBE MF, CEREZO M, et al. An arabidopsis T-DNA mutant affected in Nrt2 genes is impaired in nitrate uptake. FEBS Lett $2001 ; 489: 220-4$.

12. TONG Y, ZHOU J-J, LIZ, MILLER AJ. A twocomponent high-affinity nitrate uptake system in barley. Plant / $2005 ; 41$ : 442-50.

13. OKAMOTO M, VIDMAR JJ, GLASS AD. Regulation of NRT1 and NRT2 gene families of Arabidopsis thaliana/ responses to nitrate provision. Plant Cell Physiol 2003 ; 44 : 304-17.

14. OKAMOTO M, KUMAR A, LI W, et al. Highaffinity nitrate transport in roots of Arabidopsis thaliana depends on expression of the NAR2like gene AtNRT3.1. Plant Physiol $2006 ; 140$ : 1036-46.

15. ORSEL M, CHOPIN F, LELEU O, et al. Characterization of a two-component high-affinity nitrate uptake system in arabidopsis. Physiology and Protein-Protein interaction. Plant Physiol 2006 ; 142 : 1304-17.

16. REMANS T, NACRY P, PERVENT $M$, et al. A central role for the nitrate transporter NRT2.1 in the integrated morphological and physiological responses of the root system to $\mathrm{N}$ limitation in Arabidopsis thaliana. Plant Physiol 2006; 140 : 909-21.

17. DELROT $S$, ROCHAT C, TEGEDER M FROMMER WB. Amino acid transport. In: Lea P, Morot-Gaudry JF, eds. Plant. Nitrogen. INRA Springer Verlag, $2001: 213-35$.

18. BROUQUISSER, MASCLAUX C, FELLER U, RAYMOND P. Protein hydrolysis and nitrogen remobilisation in plant life and senescence. In : Lea P, Morot-Gaudry JF, eds. Plant. Nitrogen. INRA Springer Verlag, $2001: 275-93$.

19. MASCLAUX C, VALADIER MH, BRUGIERE N, MOROT-GAUDRY JF, HIREL B. Characterization of the sink/source transition in tobacco (Nicotiana tabacum $\mathrm{L}$.) shoots in relation to nitrogen management and leaf senescence. Planta $2000 ; 211: 510-8$ 
20. MASCLAUX-DAUBRESSE C, CARRAYOL E, VALADIER MH. The two nitrogen mobilisationand senescence-associated CS1 and GDH genes are controlled by $\mathrm{C}$ and $\mathrm{N}$ metabolites. Planta $2005 ; 221: 580-8$.

21. PAGEAU K, REISDORF-CREN M, MOROTGAUDRY JF, MASCLAUX-DAUBRESSE C. The two senescence-related markers GS1 (cytosolic glutamine synthetase) and GDH (glutamate dehydrogenase), involved in nitrogen mobilisation, are differentially regulated during pathogen attack, by stress hormones and reactive oxygen species in Nicotiana tabacum L. leaves. J Exp Bot 2006 ; 57 : 547-57.

22. LOUDET O, CHAILLOU S, CAMILLERI $C$ BOUCHEZ D, DANIEL-VEDELE F. Bay-0xShahdara recombinant inbred line population : a powerful tool for the genetic dissection of complex traits in Arabidopsis. Theor Appl Genet 2002 ; 104 : 1173 84.

23. DIAZ C, SALIBA-COLOMBANIV, LOUDET O, et al. Leaf Yellowing and anthocyanin accumulation are two genetically independent strategies in response to nitrogen limitation in Arabidopsis thaliana. Plant Cell Physiol 2006; 47 : 74-83.

24. DIAZC, PURDYS, CHRISTA, MOROTGAUDRY J-F, WINGLER A, MASCLAUXDAUBRESSE C. Characterization of markers to determine the extent and variability of leaf senescence in Arabidopsis. A metabolic profiling approach. Plant Physiol $2005 ; 138$ : 898-908.

25. MALAGOLIP, LAINE $P$, LE DEUNFFE, ROSSATO L, NEY B, OURRY A. Modeling N uptake in Brassica napus L.cv. Capitol during the growth cycle using influx kinetics of nitrate transport systems and field experimental data. Plant Physiol 2004 ; 134 : 388-400.

26. MALAGOLI P, LAINE P, ROSSATO L, OURRY A. Dynamics of nitrogen uptake and mobilization in field-grown winter oilseed rape (Brassica napus) from stem extension to harvest. I. Global $\mathrm{N}$ flows between vegetative and reproductive tissues in relation to leaf fall and their residual N. Ann Bot (Lond) 2005 ; 95 : 853-61.

27. MALAGOLI P, LAINE P, ROSSATO L, OURRY A. Dynamics of nitrogen uptake and mobilization in field-grown winter oilseed rape (Brassica napus) from stem extension to harvest. II. An ${ }^{15} \mathrm{~N}$ labelling based simulation model of $\mathrm{N}$ partitioning between vegetative and reproductive tissues. Ann Bot (Lond) 2005 ; 95 : 1187-98. 